\title{
BLOW-UP OF WEAK SOLUTIONS FOR THE SEMILINEAR WAVE EQUATIONS WITH NONLINEAR BOUNDARY AND INTERIOR SOURCES AND DAMPING
}

Abstract. We focus on the blow-up in finite time of weak solutions to the wave equation with interior and boundary nonlinear sources and dissipations. Our central interest is the relationship of the sources and damping terms to the behavior of solutions. We prove that under specific conditions relating the sources and the dissipations (namely $p>m$ and $k>m$ ), weak solutions blow up in finite time.

1. Introduction. Let $\Omega \subset \mathbb{R}^{n}$ be a bounded domain with sufficiently smooth boundary $\Gamma$. We consider the following model of semilinear wave equation with nonlinear boundary/interior monotone dissipations and nonlinear boundary/interior sources:

$$
\begin{cases}u_{t t}+g_{0}\left(u_{t}\right)=\Delta u+u|u|^{p-1} & \text { in } \Omega \times[0, \infty), \\ \partial_{\nu} u+u+g\left(u_{t}\right)=u|u|^{k-1} & \text { in } \Gamma \times[0, \infty), \\ u(0)=u_{0} \in H^{1}(\Omega) \quad \text { and } \quad u_{t}(0)=u_{1} \in L_{2}(\Omega),\end{cases}
$$

where $g_{0}(s), g(s)$ are continuous, increasing functions which model interior and boundary damping in the system.

In this paper, we are interested in the blow-up in finite time phenomenon and we investigate the relationship between the source-damping interaction and the behavior of solutions.

This general topic has attracted a lot of attention in recent years ([14, $30,36,33,27,40,29]$ and references therein). However, the majority of the

2000 Mathematics Subject Classification: Primary 35L05; Secondary 35B33, 35B35, $35 \mathrm{~L} 20$.

Key words and phrases: wave equation, nonlinear interior and boundary damping, interior and boundary supercritical sources, blow-up, nonexistence. 
work has been done either in $\mathbb{R}^{n}$ or with zero Dirichlet boundary conditions. In such cases, boundary conditions do not interfere with the equation, and boundary traces are not accounted for. Nevertheless, boundary conditions of Neumann type with "energy building" supercritical sources are natural conditions often present in interactive systems where the wave equation is coupled with other dynamics, such as structural acoustic problems or fluidstructure interactions. The action-interaction takes place on the boundary and thus the correct boundary conditions are of Neumann type [18]. These examples are not only interesting mathematically, but also have value in industrial applications. It is well known that the presence of Neumann boundary data conditions completely changes the nature of the problem, also due to the fact that the Lopatinskiı condition no longer holds (unlike for Dirichlet boundary data). The regularity theory is geometry dependent, even in the linear case. Thus, the analysis of the problem requires new methods which account for the geometry of the domain. In fact, the problem of boundary stability of wave equations has attracted considerable attention in recent years. It is well known by now that an oscillatory wave equation can be stabilized from the boundary alone, or even portion of the boundary, provided suitable geometric conditions are imposed $([17,16,22,18,12,9,20]$ and many other references). Thus questions such as: (i) how the source located on the boundary interacts with the damping; (ii) whether the boundary damping can prevent or delay the onset of finite time blow-up, are both timely and challenging.

The model considered in this paper accounts for both interior and boundary damping-sources interactions. However, the main emphasis is put on the presence of boundary dissipation. The difficulty of the problem is compounded by the fact that the sources considered can be quite "rough" by exceeding critical values of Sobolev's exponents. More specifically, in line with Sobolev's embedding $H^{1}(\Omega) \rightarrow L_{6}(\Omega)$ (in three dimensions), we can classify the interior source $f$ as follows (we consider here the most relevant case $\operatorname{dim}(\Omega)=n=3$, but the classification and analysis can be done for any other value of $n$ ):

(1) Subcritical: $1 \leq p<3$ and Critical: $p=3$. In these cases, $f$ is locally Lipschitz from $H^{1}(\Omega)$ into $L_{2}(\Omega)$.

(2) Supercritical: $3<p<5$. Then $f$ is no longer locally Lipschitz. However, the potential well energy associated with $f$, i.e. $\int_{\Omega} \hat{f}(u) d x$, where $\hat{f}$ is the antiderivative of $f$, is still well defined on the finite energy space.

(3) Super-supercritical: $5 \leq p<6$. Potential energy may not be defined on the finite energy space and thus the sources are no longer within the framework of potential well theory. 
Similarly, we can classify the boundary sources with respect to the "criticality" of Sobolev's embedding $H^{1 / 2}(\Gamma) \rightarrow L_{4}(\Gamma)$ :

(1) Subcritical: $1 \leq k<2$ and Critical: $k=2$.

(2) Supercritical: $2<k<3$.

(3) Super-supercritical: $3 \leq k<4$.

In order to discuss finite time blow-up of solutions, it is natural to start with an existence theory that includes local existence and uniqueness of finite energy solutions. This is discussed below in Section 2 where a number of relevant results are collected.

2. Existence theory. In this section we present a model that is more general than the original system (1). In fact, our equation (1) is a particular case of the semilinear wave equation

$$
\begin{cases}u_{t t}+g_{0}\left(u_{t}\right)=\Delta u+f(u) & \text { in } \Omega \times[0, \infty), \\ \partial_{\nu} u+u+g\left(u_{t}\right)=h(u) & \text { in } \Gamma \times[0, \infty), \\ u(0)=u_{0} \in H^{1}(\Omega) \quad \text { and } & u_{t}(0)=u_{1} \in L_{2}(\Omega),\end{cases}
$$

where the sources and the interior damping have more general nonlinearities (they are not necessarily of polynomial structure). The functions $g_{0}, g, f$, and $h$ represent Nemytskii operators associated with scalar, continuous realvalued functions $g_{0}(s), g(s), f(s)$, and $h(s)$. The functions $g_{0}(s)$ and $g(s)$ model the interior and boundary dissipations in the equation. They are assumed to be increasing and polynomially bounded above and below at infinity, i.e. for $|s|>1, l_{m}|s|^{m+1} \leq g_{0}(s) s \leq L_{m}|s|^{m+1}$ and $m_{q}|s|^{q+1} \leq$ $g(s) s \leq M_{q}|s|^{q+1}$. Without loss of generality, we shall assume that $q, m \geq 1$. The functions $f(s)$ and $h(s)$ represent the modeling of the sources. In order to classify the sources and briefly describe previous results, assume that $f$ and $h$ are polynomially bounded at infinity, i.e. $|f(s)| \leq|s|^{p}$ and $|h(s)| \leq|s|^{k}$ for $|s|>1$.

Equation (2) belongs to a class of problems characterized by the competing natures of the sources and the dampings. While the damping term is usually considered in the context of stability (in time) of solutions, in the case of strong nonlinearities present in the system, the damping plays a critical role in establishing even local existence of solutions.

We begin with the definition of weak solution:

Definition 2.1. By a weak solution of (2), defined on some interval $(0, T)$, we mean a function $u \in C_{w}\left(0, T ; H^{1}(\Omega)\right)$ with $u_{t} \in C_{w}\left(0, T ; L_{2}(\Omega)\right)$ such that

(a) $u_{t} \in L_{m+1}(0, T ; \Omega),\left.u_{t}\right|_{\Gamma} \in L_{q+1}(0, T ; \Gamma)$. 
(b) For all $\phi \in C\left(0, T ; H^{1}(\Omega)\right) \cap C^{1}\left(0, T ; L_{2}(\Omega)\right) \cap L_{m+1}(0, T ; \Omega)$ with $\left.\phi\right|_{\Gamma} \in L_{q+1}(0, T ; \Gamma)$,

$\int_{0}^{T} \int_{\Omega}\left(-u_{t} \phi_{t}+\nabla u \nabla \phi\right) d \Omega d t+\int_{0}^{T} \int_{\Omega} a g_{0}\left(u_{t}\right) \phi d \Omega d t+\int_{0}^{T} \int_{\Gamma} u \phi d \Gamma d t$ $=-\left.\int_{\Omega} u_{t} \phi d \Omega\right|_{0} ^{T}+\int_{0}^{T} \int_{\Gamma} h(u) \phi d \Gamma d t-\int_{0}^{T} \int_{\Gamma} g\left(u_{t}\right) \phi d \Gamma d t+\int_{0}^{T} \int_{\Omega} f(u) \phi d \Omega d t$.

(c) $\lim _{t \rightarrow 0}\left(u(t)-u_{0}, \phi\right)_{H^{1}(\Omega)}=0$ and $\lim _{t \rightarrow 0}\left(u_{t}(t)-u_{1}, \phi\right)_{L_{2}(\Omega)}=0$ for all $\phi \in C\left(0, T ; H^{1}(\Omega)\right) \cap C^{1}\left(0, T ; L_{2}(\Omega)\right) \cap L_{m+1}(0, T ; \Omega)$ with $\left.\phi\right|_{\Gamma} \in L_{q+1}(0, T ; \Gamma)$.

Here $C_{w}(0, T ; Y)$ denotes the space of weakly continuous functions with values in a Banach space $Y$.

For subcritical/critical interior sources $f$ (i.e. $p \leq 3$ ) without any boundary sources (i.e. $h=0$ ), local wellposedness is standard, and it follows from monotone operator theory $[2,22]$. Instead, the problem is more subtle when (i) interior source is (super-) supercritical (i.e. $p>3$ ), and/or (ii) a nonlinear boundary source $h(u)$ is present in the model. The presence of the damping in the system becomes essential, even at the level of local theory. This has been recognized in the literature, first in the case of interior sources [14, 36, 40,3], and more recently in the case of boundary sources $[22,39,9,5,7]$.

For supercritical interior sources, [13] and [3] exhibited existence, but no uniqueness, of weak solutions for a bounded domain $\Omega$, under the restriction $p<6 m /(m+1)$, while in [36] the same results were obtained for $\Omega=\mathbb{R}^{3}$, and compactly supported initial data, with $p<6 m /(m+1)$. In this case, it was shown additionally in [31] that if the interior damping is absent or linear, the exponent $p$ may be supercritical, i.e. $p<5$.

The treatment of boundary sources $h(u)$ is much more subtle. The main difficulty is due to the fact that the Lopatinskiur condition does not hold for the Neumann problem, i.e. the linear map $h \mapsto U(t)=\left(u(t), u_{t}(t)\right)$ where $U(t)$ is the basic Neumann solver for the wave equation (i.e., solution $U=\left(u, u_{t}\right)$ with $\left.g_{0}=0, g=0, f=0\right)$ is not bounded from $L_{2}(\Sigma)$, where $\Sigma=\Gamma \times[0, T]$, to $H^{1}(\Omega) \times L_{2}(\Omega)$, unless the dimension of $\Omega$ is equal to 1 or the initial data are compactly supported $[37,23,1]$. In fact, the maximal amount of regularity that one obtains is in general $H^{2 / 3}(\Omega) \times H^{-1 / 3}(\Omega)$ $[24,25,38]$-hence there is a "loss" of $1 / 3$ derivative, unless the boundary is flat, in which case the loss is $1 / 4$ derivative. Thus even in the subcritical case, when $h$ is locally Lipschitz $H^{1 / 2}(\Gamma) \rightarrow L_{2}(\Gamma)$, this Lipschitz property, due to the loss of $1 / 3$ derivative for the Neumann-wave map, does not translate into Lipschitz behavior of the corresponding wave map $[21,23,38]$. So even in the subcritical case, the analysis requires 
special treatment that involves an interaction with the damping (unlike the interior case). One way to deal with this issue is to start with initial data sufficiently small. In this case, potential well theories guarantee appropriately "small" solutions [28], [29], [39]. In order to deal with initial data of an arbitrary size, the idea (coming from recent developments in boundary control theory) was to exploit the boundary dissipation as a sort of "regularization" [26] offsetting singularities brought by nonhomogeneous Neumann data. In fact, this philosophy was pursued in [22], where it was shown that finite energy solutions do exist locally for locally Lipschitz functions $f$ and $h$ and for any dissipation $g$ that is continuous, monotone, and linearly bounded at infinity. Then [39] provided a full analysis of existence of finite energy solutions for the purely boundary case. With damping and source of polynomial structures: $h(u)=|u|^{k-1} u$, $g(u)=|u|^{q-1} u$, in [39] local existence of weak solutions was obtained provided that $1 \leq k<3$ (up to the supercritical case) and with the following relationship between the exponents of the damping and the source: $k<4 q /(q+1)$. The proof in [39] relies on Schauder's fixed point theorem, which requires certain compactness properties exhibited by weak solutions and excludes super-supercritical exponents in the nonlinear sources considered.

More recently, local existence and uniqueness of finite energy (i.e. $H^{1}(\Omega)$ $\times L_{2}(\Omega)$ ) solutions associated with the equation (2) were studied in [5], [7]. In comparison to previous literature, techniques of $[5,7]$ completely avoid the use of compactness and prove that the exclusion of super-supercritical boundary sources in [39] is inherent to the method, and not to the problem. Inspired by [22], the theory of nonlinear semigroups generated by maximal monotone operators was applied to a special approximation of the model. As a result, existence of local solutions for all supercritical and supersupercritical sources $(5 \leq p<6,3 \leq k<4)$ was shown, and uniqueness of finite energy solutions (a recognized open problem) was established. The relevant result is formulated below.

Assumption 1. With reference to system (2), assume

(1) Damping: $g, g_{0}$ are increasing continuous functions such that $g(0)$ $=g_{0}(0)=0$. In addition, the following growth conditions at infinity hold: There exist positive constants $m_{q}, M_{q}, l_{m}, L_{m}$ such that for $|s|>1, m_{q}|s|^{q+1} \leq g(s) s \leq M_{q}|s|^{q+1}$ and $l_{m}|s|^{m+1} \leq g_{0}(s) s \leq$ $L_{m}|s|^{m+1}$ with $q, m \geq 1$.

(2) Sources

- Internal source: $f \in C^{1}(\mathbb{R})$ and the following growth condition holds for $|s| \geq 1$ : 


$$
\begin{aligned}
& \text { - }\left|f^{\prime}(s)\right| \leq C|s|^{p-1} \text { for } 1 \leq p \leq 3, \\
& \text { - } f \in C^{2}(\mathbb{R}),\left|f^{\prime \prime}(s)\right| \leq C|s|^{p-2} \text { for } 3<p<6 m /(m+1),
\end{aligned}
$$

- Boundary source: We distinguish sublinear $(q<1)$ and superlinear $(q \geq 1)$ damping.

- In the sublinear case: $h \in C^{1}(\mathbb{R}),\left|h^{\prime}(s)\right| \leq C\left[|s|^{k-1}+1\right], 0<$ $q<1,1 \leq k \leq 4 q /(q+1)$.

- In the superlinear case: $h \in C^{2}(\mathbb{R}),\left|h^{\prime \prime}(s)\right| \leq C\left[1+|s|^{k-2}\right]$, $2 \leq k \leq 4 q /(q+1)$.

Let $H \equiv H^{1}(\Omega) \cap L_{r}(\Omega) \cap L_{s}(\Gamma) \times L_{2}(\Omega)$, where $r=\frac{3}{2}(p-1)$ and $s=2(k-1)$. By $L_{s}(\Gamma)$ we mean functions in $H^{1}(\Omega)$ whose traces on the boundary are in $L_{s}(\Gamma)$. Note that for $p \leq 5, H^{1}(\Omega) \subset L_{r}(\Omega)$, and for $k \leq 3$, $H^{1}(\Omega) \subset L_{s}(\Gamma)$.

TheOREM 2.2 (Existence and uniqueness). Let $H \equiv H^{1}(\Omega) \cap L_{r}(\Omega) \cap$ $L_{s}(\Gamma) \times L_{2}(\Omega)$, where $r=\frac{3}{2}(p-1)$ and $s=2(k-1)$.

- Local existence: With reference to equation (2) and under Assumption 1 above, there exists a local in time weak solution $U=\left(u, u_{t}\right) \in$ $C\left(\left[0, T_{M}\right), H\right)$, where the maximal time of existence $T_{M}$ depends on the initial data $|U(0)|_{H}$, locally Lipschitz constants, and $m_{q}, l_{m}$. The said solution is unique.

- Global existence: Under the additional assumption that $p \leq m$, $k \leq q$, the local solution becomes global.

We summarize these results with the following illustrations:

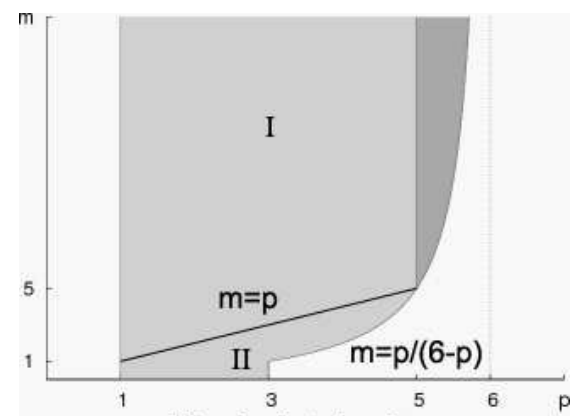

Fig. A - Interior Case

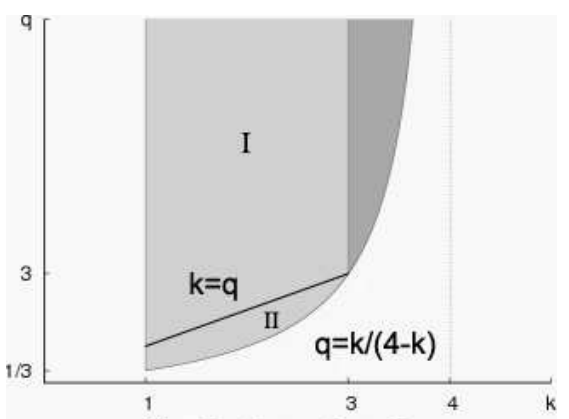

Fig. B - Boundary Case

(1) Local existence holds in the interior case [Fig. A] for the entire region $1 \leq p \leq 3$ or $p \leq 6 m /(m+1)$ (or $m \geq p /(6-p)$ ) if $p>3$ and in the boundary case [Fig. B] for the whole region described by $1 \leq k \leq$ $4 q /(q+1)$ (or $q \geq k /(4-k)$ ) [5]. The dark shaded region indicates the range of parameters $p$ and $k$ corresponding to super-supercritical cases. The existence theory for the parameters delimited by light shaded subregions (that includes supercritical values of $p, k, p<5, k<3$ ), with the sources of 
a polynomial structure, was established in earlier literature $([39,36,13,3,9]$ and references therein).

(2) Uniqueness. While uniqueness of weak solutions in the interior-subcritical case is standard, it has been a longstanding open issue [13, 36, 9] in the supercritical cases with both boundary and interior sources. This question has recently been resolved in [7].

(3) Parameters corresponding to global existence ( $m \geq p$ and $q \geq k$ ) are given by region I in Fig. A, respectively Fig. B, while blow-up in finite time holds in complementary region II (precise description is given in Theorems 3.1 and 3.2 ).

The result presented in Theorem 2.2 provides local existence and uniqueness of weak solutions. Such solutions, without further hypotheses, may not be global, as they may blow up in finite time. The phenomenon of blow-up depends on the interaction between the source and the damping. Damping has a tendency to extend the span life of solutions. This fact was realized first in [14], in the case of interior source-damping interaction. Our main task in this paper is to analyze this interaction in the context of interior and boundary interactions. As we mentioned before, our main result pertains to finite time blow-up of weak solutions. We shall prove that in the region of parameters complementary to the region associated with global existence theory, weak solutions of negative energy blow up in finite time. In analyzing the problem, we shall consider two sources contributing to potential blow-up: interior and boundary sources, along with two dissipative mechanisms, one in the interior and one on the boundary. The tendency of the latter is to prevent or delay the onset of blow-up. Our main result is formulated in Section 3 below.

3. Blow-up of solutions in finite time. Below we present the main result of this paper. We shall use the following notation: $\|u\|_{s, \Omega}=|u|_{H^{s}(\Omega)}$, $\|u\|$ refers to the case $s=0,\|u\|_{p+1}=|u|_{L_{p+1}(\Omega)}$. Notation with single vertical bars refers to spaces on the boundary. Since $\|\nabla u\|^{2}+|u|^{2}$ is a norm equivalent to the $H^{1}(\Omega)$ norm, we shall topologize $H^{1}(\Omega)$ with respect to that norm.

With model (1) we associate the following assumptions:

Assumption 2.

(i) $g(s)$ is an increasing continuous function such that $g(0)=0$. In addition, the following growth condition at infinity is satisfied: there exists $q \geq 1$ and positive constants $m_{q}$ and $M_{q}$ such that for $|s|>1$, $m_{q}|s|^{q+1} \leq g(s) s \leq M_{q}|s|^{q+1}$.

(ii) $p>m$. 
(iii) Sobolev embedding restrictions: $p+1 \leq 2 n /(n-2)$ and $k+1 \leq$ $(2 n-2) /(n-2)$.

(iv) either $k>q$ or $p>2 q-1$.

TheOREM 3.1 (Critical and supercritical exponents-Finite time blowup). Assume the validity of Assumption 2 and in addition assume that $E(0)<0$, where $E(0)$ is the initial energy given by

$$
E(0)=\frac{1}{2}\left(\left\|u_{1}\right\|^{2}+\left\|u_{0}\right\|_{1, \Omega}^{2}\right)-\frac{1}{p+1}\left\|u_{0}\right\|_{p+1}^{p+1}-\frac{1}{k+1}\left|u_{0}\right|_{k+1}^{k+1} .
$$

Then any weak solution to (1) blows up in finite time. By this we mean that $\|u(t)\|_{p+1}+|u(t)|_{k+1} \rightarrow \infty$ as $t \rightarrow T_{0}$, and consequently $\|u(t)\|_{1, \Omega} \rightarrow \infty$ as $t \rightarrow T_{0}$, for some $0<T_{0}<\infty$.

REMARK 1. A blow-up result for a potential well solution without damping and with $f=0$ has been established in [28]. It was also shown in [28] that for $k>1$, the finite energy $E(t)$ tends to minus infinity. However, the convexity methods of [28] do not apply to problems with damping. The result in [28] is a nonexistence result rather than a blow-up result (it is a blow-up result for potential well solutions only).

Theorem 3.1 exhibits blow-up of all local weak solutions (not necessarily from a potential well) and with the boundary-interior damping/source combination. Theorem 2.2 shows that the presence of the boundary dissipation does extend the life span of local solutions $(k \leq q)$, but the value $k=q$ is critical. On the other hand, Theorem 3.1 shows that $k>q$ with an incremental interior source $p>1$ causes solutions to blow up. Since global solutions exist in the complementary region $k \leq q$ and $p \leq m$ (see [6]), our results are sharp.

An interesting question to ask is whether the blow-up phenomena can take place for solutions of positive energy. This is known to hold for some configurations of potential well solutions $[40,41,9]$. However, the methodology employed there forces potential well type restrictions on the solutions. Instead, our work is inspired by a more direct approach developed for the interior case in $[30,14]$ (see also [3, 4]) which can be adapted to all weak solutions of negative energy with both boundary-interior damping and subsupercritical sources.

We note that Theorem 3.1 does not cover cases of super-supercritical solutions. In fact, the reason for this is that super-supercritical sources are above the Sobolev scale. For this case we have a slightly different blow-up result which attests global nonexistence of potential solutions corresponding to sufficiently negative initial energy. The corresponding result is formulated below. 
Theorem 3.2 (Super-supercritical exponents - Global nonexistence). Assume the validity of part (i) in Assumption 2 along with the condition that $p>m$ and $k>q$. Then there exists a constant $M>0$ such that every weak solution with initial energy $E(0)<-M$ blows up in finite time. By this we mean that $\|u(t)\|_{p+1}+|u(t)|_{k+1} \rightarrow \infty$ as $t \rightarrow T_{0}$, for some $0<T_{0}<\infty$.

REMARK 2. We note that for the range of parameters in Theorem 3.2 the existence result stated in Theorem 2.2 does not apply (this is due to the restrictions $p<6 m /(m+1)$ and $k \leq 4 q /(q+1)$ which are not satisfied in the super-supercritical cases with $p>m, k>q)$. Thus, the result of Theorem 3.2 is a global nonexistence result rather than a blow-up result.

REMARK 3 . The values of the parameters $M, T_{0}$ that appear in Theorem 3.2 can be estimated directly in terms of the data of the problem [32].

The rest of the paper is devoted to the proofs of the main results. The proofs rely on an adaptation of the method developed in [30, 14], which is then combined with the theory of interpolation in Besov spaces.

4. Proof of Theorem 3.1. The validity of the energy identity for all weak solutions is fundamental to the proof of blow-up of solutions. While an energy inequality can be typically shown by weak lower-semicontinuity arguments, the energy identity is much more demanding and may not hold without the knowledge of sufficient regularity of weak solutions. In the case of the model under consideration in this article, the energy identity has been shown in [7] by using an appropriate finite difference scheme. The corresponding result is reported below.

4.1. Energy identity. The first step in the proof of our result is to derive the energy identity for weak solutions of system (1). The validity of this equality depends on the presence of the damping in the system.

Lemma 4.1 ([7]). Let u be a weak solution of system (1) with the following a priori regularity:

$$
\left(u, u_{t}\right) \in B([0, T], H),
$$

where $B([0, T], H)$ is the space of $H$-valued functions which are bounded on $[0, T]$, endowed with the usual norm $|x|_{B([0, T], H)}=\sup _{t \in[0, T]}|x(t)|_{H}$, and

$$
u_{t} \in L_{m+1}(0, T ; \Omega),\left.\quad u_{t}\right|_{\Gamma} \in L_{q+1}(0, T ; \Gamma)
$$

Then the following energy identity holds for all $0 \leq s \leq t \leq T$ :

$$
E(t)+\int_{s}^{t} \int_{\Omega} g_{0}\left(u_{t}\right) u_{t} d \Omega d z+\int_{s} \int_{\Gamma}\left(g\left(u_{t}\right) u_{t} d \Gamma d z=E(s)\right.
$$


where

$E(t) \equiv \frac{1}{2}\left\|u_{t}(t)\right\|^{2}+\frac{1}{2}\|\nabla u(t)\|^{2}+\frac{1}{2}|u|^{2}-\frac{1}{p+1}\|u(t)\|_{p+1}^{p+1}-\frac{1}{k+1}|u(t)|_{k+1}^{k+1}$.

Notice that the result of Lemma 4.1 can be formally obtained by integrating by parts in time and using Green's formula. However, the procedure is only formal. Its justification requires additional smoothness of solutions, an information that is not available. For a detailed proof of the lemma, see [7], where finite difference approximation of time derivatives combined with weak continuity methods are used [15].

4.2. Lyapunov function. The main idea of the proof of Theorem 3.1 is due to $[14,30]$ with some technicalities borrowed from [3] (where the wave equation with interior degenerate damping was considered). However, the presence of boundary damping along with boundary source changes the picture. Boundary damping is another source of dissipation, though localized to the boundary only. Its effect and potential for geometric propagation need to be taken into consideration.

Proof of Theorem 3.1. With the energy function defined by

$$
E(t)=\frac{1}{2}\left(\left\|u_{t}(t)\right\|_{2}^{2}+\|u(t)\|_{H^{1}(\Omega)}^{2}\right)-\frac{1}{p+1}\|u(t)\|_{p+1}^{p+1}-\frac{1}{k+1}|u(t)|_{k+1}^{k+1}
$$

the generalized solutions satisfy the energy equality

$$
E(t)-E(0)=-\int_{0}^{t} \int_{\Omega} g_{0}\left(u_{t}(t)\right) u_{t}(t) d x d t d \Omega-\int_{0}^{t} \int_{\Gamma} g\left(u_{t}\right) u_{t}(t) d \Gamma d t .
$$

Following $[14,30]$, we let

$$
H(t)=-E(t)
$$

and we take a classical Lyapunov corrector [17]

$$
F(t)=\left(u, u_{t}\right)_{\Omega} .
$$

Define

$$
y(t)=(H(t))^{1-\alpha}+\varepsilon F(t) .
$$

The goal is to show that

$$
y^{\prime}(t) \geq c y^{\frac{1}{1-\alpha}}
$$

for some small $0<\alpha<1$ (determined later) and $c>0$.

In fact, showing that $y(t) \rightarrow \infty$ implies (after some calculations involving $F(t))$ that $H(t) \rightarrow \infty$, hence $E(t) \rightarrow-\infty$. This, in turn, implies the blowup of the potential energy. Thus, our task is to establish inequality (4) with some nonnegative $\alpha$.

From (4.2), we get

$$
y^{\prime}(t)=(1-\alpha)(H(t))^{-\alpha} H^{\prime}(t)+\varepsilon F^{\prime}(t) .
$$


First, using the absolute continuity of $H(t)$, Assumption 1 and the energy identity, one has

$$
H^{\prime}(t)=\int_{\Omega} g_{0}\left(u_{t}(t)\right) u_{t}(t) d \Omega+\int_{\Gamma} g\left(u_{t}\right) u_{t}(t) d \Gamma d t \geq 0 .
$$

Therefore,

$$
H(t) \geq H(0)=-E(0)>0 \quad \text { for } 0 \leq t \leq T .
$$

We estimate $F^{\prime}(t)$. From weak formulation of solution, after noticing that $\|u\|_{1, \Omega}^{2}$ is equivalent to $\|\nabla u\|^{2}+|u|_{0, \Gamma}^{2}$, we obtain

$$
\begin{aligned}
F^{\prime}(t)= & \left\|u_{t}(t)\right\|_{2}^{2}-\|u(t)\|_{1, \Omega}^{2}+\|u(t)\|_{p+1}^{p+1} \\
& +|u(t)|_{k+1}^{k+1}-\int_{\Omega} u g_{0}\left(u_{t}\right) d \Omega-\int_{\Gamma} g\left(u_{t}\right) u d \Gamma+C_{0} E(t)+C_{0} H(t)
\end{aligned}
$$

(since $E(t)+H(t)=0)$. Hence

$$
\begin{aligned}
F^{\prime}(t)= & \left(1+\frac{C_{0}}{2}\right)\left\|u_{t}(t)\right\|_{2}^{2}+\left(\frac{C_{0}}{2}-1\right)\|u(t)\|_{1, \Omega}^{2}+C_{0} H(t) \\
& +\left(1-\frac{C_{0}}{p+1}\right)\|u(t)\|_{p+1}^{p+1}+\left(1-\frac{C_{0}}{k+1}\right)|u(t)|_{k+1}^{k+1} \\
& -\int_{\Omega} u g_{0}\left(u_{t}\right) d \Omega-\int_{\Gamma} g\left(u_{t}\right) u d \Gamma .
\end{aligned}
$$

Selecting $C_{0}=2+\beta$ with $\beta>0$, we gain control of the superlinear terms, which in turn leads to the following proposition.

Proposition 1.

$$
\begin{aligned}
F^{\prime}(t)= & \left(2+\frac{\beta}{2}\right)\left\|u_{t}(t)\right\|_{2}^{2}+\frac{\beta}{2}\|u(t)\|_{1, \Omega}^{2}+(2+\beta) H(t) \\
& +\frac{p-1-\beta}{p+1}\|u(t)\|_{p+1}^{p+1}+\frac{k-1-\beta}{k+1}|u(t)|_{k+1}^{k+1} \\
& -\int_{\Omega} u g_{0}\left(u_{t}\right) d \Omega-\int_{\Gamma} g\left(u_{t}\right) u d \Gamma .
\end{aligned}
$$

Thus the entire effort goes into the estimates for $\int_{\Omega} u g_{0}\left(u_{t}\right) d \Omega$ and $\int_{\Gamma} g\left(u_{t}\right) u d \Gamma$. We start with the former.

LEMMA 4.2.

$$
\int_{\Omega} u g_{0}\left(u_{t}\right) d \Omega \leq C_{\delta_{1}} H^{\prime}(t)(H(t))^{-\alpha}+\delta_{1}\left[B(p, k, u)+C_{1}(H(0))\|u\|_{1, \Omega}^{2}\right] .
$$

Proof. Applying Hölder's inequality with exponents $m+1$ and $(m+1) / m$, along with the bounds imposed on $g_{0}$, we obtain 


$$
\begin{aligned}
\left|\int_{\Omega} u g_{0}\left(u_{t}\right) d \Omega\right| & \leq\|u(t)\|_{m+1}\left\|u_{t}(t)\right\|_{m+1}^{m} \\
& \leq\|u(t)\|_{p+1}\left\|u_{t}(t)\right\|_{m+1}^{m} \leq C\|u(t)\|_{p+1}\left(H^{\prime}(t)\right)^{\frac{m}{m+1}} \\
& =C\left(H^{\prime}(t)\right)^{\frac{m}{m+1}}(B(p, k, u))^{\frac{-\alpha m}{m+1}}(B(p, k, u))^{\frac{\alpha m}{m+1}}\|u(t)\|_{p+1}
\end{aligned}
$$

where we used the assumption $m \leq p,(6)$ and the notation

$$
B(p, k, u)=\frac{1}{k+1}|u(t)|_{k+1}^{k+1}+\frac{1}{p+1}\|u(t)\|_{p+1}^{p+1} \geq H(t) \geq H(0)>0 .
$$

Application of Young's inequality with exponents $(m+1) / m$ and $m+1$, and the use of (9) gives

$$
\begin{aligned}
\left|\int_{\Omega} u g_{0}\left(u_{t}\right) d \Omega\right| \leq & C_{\delta_{1}} H^{\prime}(t)(B(p, k, u))^{-\alpha} \\
& +\delta_{1}(B(p, k, u))^{\alpha m}\|u(t)\|_{p+1}^{m+1} \\
\leq & C_{\delta_{1}} H^{\prime}(t)(H(t))^{-\alpha}+\delta_{1}(B(p, k, u))^{\alpha m}\|u(t)\|_{p+1}^{m+1} .
\end{aligned}
$$

Assuming $\alpha m<1$ and using Young's inequality with exponents $1 / \alpha m$ and $1 /(1-\alpha m)$ yields

$$
(B(p, k, u))^{\alpha m}\|u(t)\|_{p+1}^{m+1} \leq c_{1} B(p, k, u)+c_{2}\|u(t)\|_{p+1}^{\frac{m+1}{1-\alpha m}} .
$$

In order to estimate $\|u(t)\|_{p+1}^{(m+1) /(1-\alpha m)}$, we further restrict $\alpha$ so that, due to the source dominating the damping $(m<p)$, we can achieve

$$
\frac{m+1}{1-\alpha m} \leq p+1 \Leftrightarrow \alpha \leq \frac{p-m}{m(p+1)}
$$

in addition to

$$
\alpha m<1 \Leftrightarrow \alpha<1 / m .
$$

At this point, we need to distinguish high and low frequencies.

CASE 1 (High frequencies): $\|u(t)\|_{p+1} \geq c_{0}$ for any (possibly small) $c_{0}>0$. Then using (12) and (13) we have

$$
\begin{aligned}
\|u(t)\|_{p+1}^{\frac{m+1}{1-\alpha m}} & =\|u(t)\|_{p+1}^{p+1}\|u(t)\|_{p+1}^{\frac{m+1}{1-\alpha m}-(p+1)} \leq C\|u(t)\|_{p+1}^{p+1} c_{0}^{\frac{m+1}{1-\alpha m}-(p+1)} \\
& \leq C B(p, k, u) c_{0}^{\frac{m+1}{1-\alpha m}-(p+1)} .
\end{aligned}
$$

CASE 2 (Small frequencies): $\|u(t)\|_{p+1} \leq c_{0}$ for suitably small $c_{0}>0$. This case involves the interaction with the boundary source, which "takes over" for small frequencies of the internal source. Indeed, when the internal source becomes "small", the boundary source "kicks in" and provides a lower bound for the $H^{1}$ norm of the solution. The above follows from the following calculations: 
We select a constant $c_{0}$ small enough so that $c_{0}^{p+1} \leq(p+1) H(0) / 2$. Hence from (9),

$$
\frac{1}{k+1}|u(t)|_{k+1}^{k+1} \geq \frac{H(0)}{2}
$$

and by Sobolev's embedding $|u|_{k+1} \leq C_{\Omega}\|u\|_{1, \Omega}$, so that we have the following lower bound for small frequencies:

$$
\|u(t)\|_{1, \Omega}^{k+1} \geq \frac{k+1}{2 C_{\Omega}} H(0) .
$$

Consider two subcases:

(i) $m+1 \geq 2(1-\alpha m)$ (so $p>1)$,

(ii) $m+1<2(1-\alpha m)$.

In the first case, since $(m+1) /(1-\alpha m) \geq 2$, and by Assumption 2 and Sobolev's embedding, we have $\|u\|_{p+1} \leq C_{\Omega}\|u\|_{H^{1}(\Omega)}$, and thus

$$
\|u(t)\|_{p+1}^{\frac{m+1}{1-\alpha m}} \leq C_{\Omega}\|u\|_{H^{1}(\Omega)}^{2}\|u\|_{p+1}^{\frac{m+1}{1-\alpha m}-2} \leq\|u\|_{H^{1}(\Omega)}^{2} c_{0}^{\frac{m+1}{1-\alpha m}-2} \leq C\|u\|_{H^{1}(\Omega)}^{2} .
$$

In the second subcase, $(m+1) /(1-\alpha m) \leq 2$ and from Assumption 2 we have

$$
\|u(t)\|_{p+1}^{\frac{m+1}{1-\alpha m}} \leq C_{\Omega}\|u(t)\|_{1, \Omega}^{2}\|u(t)\|_{1, \Omega}^{\frac{m+1}{1-\alpha m}-2} .
$$

From (15) and recalling that $(m+1) /(1-\alpha m)-2 \leq 0$, we obtain

$$
\|u(t)\|_{p+1}^{\frac{m+1}{1-\alpha m}} \leq C_{\Omega}\|u\|_{1, \Omega}^{2}\|u\|_{1, \Omega}^{\frac{m+1}{1-\alpha m}-2} \leq C(H(0))^{\frac{\frac{m+1}{1-\alpha m}-2}{k+1}}\|u\|_{1, \Omega}^{2} .
$$

Note that the exponent of $H(0)$ is negative. Let

$$
r=\frac{2-\frac{m+1}{1-\alpha m}}{k+1} \geq 0
$$

Summarizing subcases (i) and (ii), in either case 1 or 2 , we have the following estimate:

$$
\|u(t)\|_{p+1}^{\frac{m+1}{1-\alpha m}} \leq C_{\Omega}\left[\|u\|_{1, \Omega}^{2}\left(1+(H(0))^{-r}\right)+B(p, k, u)\right] .
$$

This combined with (10) leads to our final estimate for $\int_{\Omega} u g_{0}\left(u_{t}\right) \mid d \Omega$ :

$$
\int_{\Omega} u g_{0}\left(u_{t}\right) d \Omega \leq C_{\delta_{1}} H^{\prime}(t)(H(t))^{-\alpha}+\delta_{1}\left[B(p, k, u)+C_{1}(H(0))\|u\|_{1, \Omega}^{2}\right]
$$

where $\delta_{1}$ is adjusted to normalize the coefficient $B(p, k, u)$. below:

Now we have to estimate $\int_{\Gamma} g\left(u_{t}\right) u d \Gamma$. The corresponding result is given

LEMMA 4.3.

$\int_{\Gamma} g\left(u_{t}(t)\right) u(t) d \Gamma \leq C_{\delta_{2}} H^{\prime}(t)(H(t))^{-\alpha}+\delta_{2}\left[B(p, k, u)+C_{2}(H(0))\|u(t)\|_{1, \Omega}^{2}\right]$. 
Proof. Using Hölder's inequality with exponents $q+1$ and $(q+1) / q$ we obtain

$$
\begin{aligned}
\int_{\Gamma} g\left(u_{t}\right) u d \Gamma & \leq c_{0}\left(\int_{\Gamma}\left|g\left(u_{t}\right)\right|^{\frac{q+1}{q}} d \Gamma\right)^{\frac{q}{q+1}}\left(\int_{\Gamma}|u(t)|^{q+1} d \Gamma\right)^{\frac{1}{q+1}} \\
& \leq c_{0}\left(\int_{\Gamma} g\left(u_{t}\right) u_{t} d \Gamma\right)^{\frac{q}{q+1}}\left(\int_{\Gamma}|u(t)|^{q+1} d \Gamma\right)^{\frac{1}{q+1}} \\
& \leq C\left(H^{\prime}(t)\right)^{\frac{q}{q+1}}|u(t)|_{q+1} \\
& =C\left(H^{\prime}(t)\right)^{\frac{q}{q+1}}(B(p, k, u))^{\frac{-\alpha q}{q+1}}(B(p, k, u))^{\frac{\alpha q}{q+1}}|u(t)|_{q+1}
\end{aligned}
$$

where we have used Assumption 1, $B(p, k, u)$ defined in (9), and $\alpha$ satisfying (13), and (12).

Now applying Young's inequality with exponents $(q+1) / q$ and $q+1$, using (9), and recalling that $k \geq q$ (the case $p \geq 2 q-1$ will be treated subsequently), we obtain

$$
\begin{aligned}
\left|\int_{\Gamma} g\left(u_{t}\right) u d \Gamma\right| & \leq C_{\delta_{2}} H^{\prime}(t)(B(p, k, u))^{-\alpha}+\delta_{2}(B(p, k, u))^{\alpha q}|u(t)|_{k+1}^{q+1} \\
& \leq C_{\delta_{2}} H^{\prime}(t)(H(t))^{-\alpha}+\delta_{2}(B(p, k, u))^{\alpha q}|u(t)|_{k+1}^{q+1} .
\end{aligned}
$$

In addition to (13) and (12), we also assume $\alpha q<1$. Then using Young's inequality with exponents $1 / \alpha q$ and $1 /(1-\alpha q)$, we get

$$
(B(p, k, u))^{\alpha q}|u(t)|_{k+1}^{q+1} \leq c_{1} B(p, k, u)+c_{2}|u(t)|_{k+1}^{\frac{q+1}{1-\alpha q}} .
$$

In order to estimate $|u(t)|_{k+1}^{(q+1) /(1-\alpha q)}$, we further restrict $\alpha$ so that

$$
(q+1) /(1-\alpha q) \leq k+1 \Leftrightarrow \alpha \leq \frac{k-q}{q(k+1)}
$$

in addition to

$$
\alpha q<1 \Leftrightarrow \alpha<1 / q .
$$

At this point, we need to distinguish "large" and "small" frequencies:

CASE 1: $|u(t)|_{k+1} \geq c_{0}$ for any $c_{0}>0$. Then using (19) we have

$$
\begin{aligned}
|u(t)|_{k+1}^{\frac{q+1}{1-\alpha q}} & =|u(t)|_{k+1}^{k+1}|u(t)|_{k+1}^{\frac{q+1}{1-\alpha q}-(k+1)} \leq C|u(t)|_{k+1}^{k+1} c_{0}^{\frac{q+1}{1-\alpha q}-(k+1)} \\
& \leq C B(p, k, u) c_{0}^{\frac{q+1}{1-\alpha q}-(k+1)} .
\end{aligned}
$$

CASE 2: $|u(t)|_{k+1} \leq c_{0}$ for suitably small $c_{0}>0$ which will later be taken as $c_{0} \leq(k+1) H(0) / 2$. If $(q+1) /(1-\alpha q) \geq 2$ and remembering Assumption 2, we know that $|u|_{k+1} \leq C_{\Omega}\|u\|_{1, \Omega}$ and thus

$$
|u(t)|_{k+1}^{\frac{q+1}{1-\alpha q}} \leq C_{\Omega}\|u\|_{1, \Omega}^{2}|u|_{k+1}^{\frac{q+1}{1-\alpha q}-2} \leq\|u\|_{1, \Omega}^{2} c_{0}^{\frac{q+1}{1-\alpha q}-2} \leq C\|u\|_{1, \Omega}^{2} .
$$


Otherwise, if $(q+1) /(1-\alpha q) \leq 2$ and from Assumption 2 we have

$$
|u(t)|_{k+1}^{\frac{q+1}{1-\alpha q}} \leq C_{\Omega}\|u\|_{1, \Omega}^{2}\|u\|_{1, \Omega}^{\frac{q+1}{1-\alpha q}-2}
$$

Selecting $c_{0}$ small enough, $c_{0} \leq(k+1) H(0) / 2$, and using (9) we can achieve

$$
\frac{1}{p+1}\|u\|_{p+1}^{p+1} \geq \frac{H(0)}{2} .
$$

Hence by Sobolev's embedding $(p+1 \leq 2 n /(n-2))$ we have

$$
\|u\|_{1, \Omega}^{p+1} \geq C_{\Omega} H(0)>0 .
$$

Inserting (21) and (20) into (4.2) we get

$$
|u(t)|_{k+1}^{\frac{q+1}{1-\alpha q}} \leq C_{p}(H(0))^{\frac{1}{p+1}\left(\frac{q+1}{1-\alpha q}-2\right)}\|u\|_{1, \Omega}^{2} .
$$

Set

$$
s=\frac{1}{p+1}\left(2-\frac{q+1}{1-\alpha q}\right)>0
$$

Then (22) becomes

$$
|u(t)|_{k+1}^{\frac{q+1}{1-\alpha q}} \leq C_{p}(H(0))^{-s}\|u\|_{1, \Omega}^{2} .
$$

Thus in either case we obtain

$$
|u(t)|_{k+1}^{\frac{q+1}{1-\alpha q}} \leq C_{\Omega}\left[B(p, k, u)+\left((H(0))^{-s}+1\right)\|u\|_{1, \Omega}^{2}\right] .
$$

This leads to our final estimate for $\int_{\Gamma} g\left(u_{t}\right) u d \Gamma$ (see (18)):

$$
\int_{\Gamma} g\left(u_{t}\right) u d \Gamma \leq C_{\delta_{2}} H^{\prime}(t)(H(t))^{-\alpha}+\delta_{2}\left[B(p, k, u)+C_{2}(H(0))\|u\|_{1, \Omega}^{2}\right]
$$

where $\delta_{2}$ was adjusted in order to absorb the coefficient of $B(p, k, u)$.

Completion of the proof of Theorem 3.1. Combining the estimates of Lemmas 4.2 and 4.3 along with (7) we obtain the final estimate for $F^{\prime}(t)$ :

$$
\begin{aligned}
F^{\prime}(t) \geq & (2+\beta / 2)\left\|u_{t}(t)\right\|^{2}+\left(\beta / 2-\delta_{1} C_{1}-\delta_{2} C_{2}\right)\|u(t)\|_{1, \Omega}^{2} \\
& +(2+\beta) H(t)+\left(\lambda-\delta_{1}-\delta_{2}\right) B(p, k, u)-C_{\delta} H^{\prime}(t)(H(t))^{-\alpha}
\end{aligned}
$$

where

- $\lambda=\min \left\{\frac{p-1-\beta}{p+1}, \frac{k-1-\beta}{k+1}\right\}>0$ for sufficiently small $\beta$,

- $\alpha<\min \left\{\frac{1}{m}, \frac{1}{q}, \frac{p-m}{m(p+1)}, \frac{k-q}{q(k+1)}\right\}$,

- $C_{\delta}=\max \left\{C_{\delta_{1}}, C_{\delta_{2}}\right\}$. 
Inserting (23) back into (5) we obtain

$$
\begin{aligned}
y^{\prime}(t) \geq & (1-\alpha) H^{\prime}(t)(H(t))^{-\alpha}+\varepsilon(2+\beta / 2)\left\|u_{t}(t)\right\|^{2} \\
& +\varepsilon\left(\beta / 2-\delta_{1} C_{1}-\delta_{2} C_{2}\right)\|u(t)\|_{1, \Omega}^{2}+\varepsilon(2+\beta) H(t) \\
& +\varepsilon\left(\lambda-\delta_{1}-\delta_{2}\right) B(p, k, u)-\varepsilon C_{\delta_{1}, \delta_{2}} H^{\prime}(t)(H(t))^{-\alpha} .
\end{aligned}
$$

Taking $\delta_{1}$ and $\delta_{2}$ small enough such that $\delta_{1} C_{1}+\delta_{2} C_{2}<\beta / 4$ and $\delta_{1}+\delta_{2}<$ $\lambda / 2$, we achieve

Proposition 2.

$$
\begin{aligned}
y^{\prime}(t) \geq & {\left[(1-\alpha)-\varepsilon C_{\delta}\right] H^{\prime}(t)(H(t))^{-\alpha}+\varepsilon(2+\beta / 2)\left\|u_{t}(t)\right\|^{2} } \\
& +\varepsilon(\beta / 4)\|u(t)\|_{1, \Omega}^{2}+(\lambda \varepsilon / 2) B(p, k, u)+\varepsilon(2+\beta) H(t) .
\end{aligned}
$$

For sufficiently small $\varepsilon,(24)$ shows that, in particular

$$
y^{\prime}(t) \geq c H(t)>0, \quad y(0)>0 .
$$

We would like to estimate $H(t)$ in terms of $y^{1 / 1-\alpha}$ and other positive terms in the inequality stated in Proposition 2. To this end, we estimate

$$
\begin{aligned}
(F(t))^{\frac{1}{1-\alpha}} & \leq\|u(t)\|^{\frac{1}{1-\alpha}}\left\|u_{t}(t)\right\|^{\frac{1}{1-\alpha}} \leq c\left\|u_{t}(t)\right\|^{2}+C\|u(t)\|^{\frac{2}{1-2 \alpha}} \\
& \leq c\left\|u_{t}\right\|^{2}+C\left(\int_{\Omega}|u|^{p+1} d x\right)^{\frac{2}{(p+1)(1-2 \alpha)}} .
\end{aligned}
$$

Here we have used Young's inequality with exponents $2(1-\alpha)$ and $\frac{2(1-\alpha)}{1-2 \alpha}$ and the fact that $p+1>2$.

We shall impose the restriction that

$$
(p+1)(1-2 \alpha) \geq 2 \Leftrightarrow \alpha \leq \frac{p-1}{2(p+1)} .
$$

Now again we consider "large" and "small" frequencies, so we have the following cases:

CASE 1: $\|u\|_{p+1}^{p+1} \geq c_{0}=1$. Then

$$
\begin{aligned}
\left(\int_{\Omega}|u|^{p+1} d x\right)^{\frac{2}{(p+1)(1-2 \alpha)}} & =\int_{\Omega}|u|^{p+1} d x \cdot\left(\int_{\Omega}|u|^{p+1} d x\right)^{\frac{2}{(p+1)(1-2 \alpha)}-1} \\
& \leq \int_{\Omega}|u|^{p+1} d x \cdot c_{0}^{\frac{2}{(p+1)(1-2 \alpha)}-1} .
\end{aligned}
$$

CASE 2: $\|u\|_{p+1}^{p+1} \leq c_{0}=1$. Then

$$
\|u\|_{p+1}^{\frac{2}{1-2 \alpha}}=\|u\|_{p+1}^{2}\|u\|_{p+1}^{\frac{4 \alpha}{1-2 \alpha}} \leq C\|u\|_{1, \Omega}^{2}\|u\|_{p+1}^{\frac{4 \alpha}{1-2 \alpha}} \leq C\|u\|_{1, \Omega}^{2} .
$$

Thus in both cases we have

$$
(F(t))^{\frac{1}{1-\alpha}} \leq C\left[\left\|u_{t}\right\|^{2}+\|u\|_{1, \Omega}^{2}+\int_{\Omega}|u|^{p+1}\right]
$$


which means

$$
\begin{aligned}
H(t) & \geq c y^{\frac{1}{1-\alpha}}-C \varepsilon^{\frac{1}{1-\alpha}}(F(t))^{\frac{1}{1-\alpha}} \\
& \geq c y^{\frac{1}{1-\alpha}}-C \varepsilon^{\frac{1}{1-\alpha}}\left[\left\|u_{t}\right\|^{2}+\|u\|_{1, \Omega}^{2}+B(p, k, u)\right] .
\end{aligned}
$$

Inserting (28) back into (24) and selecting suitably small $\varepsilon$ gives us the desired inequality

$$
y^{\prime}(t) \geq c y^{\frac{1}{1-\alpha}}, \quad y(0)>0,
$$

whose solution blows up in finite time. Now recall from (28) that

$$
\begin{aligned}
c(y(t))^{\frac{1}{1-\alpha}} & \leq H(t)+C \varepsilon^{\frac{1}{1-\alpha}}\left[\left\|u_{t}\right\|^{2}+\|u\|_{1, \Omega}^{2}+B(p, k, u)\right] \\
& =\left(1+C \varepsilon^{\frac{1}{1-\alpha}}\right) B(p, k, u)+\left(C \varepsilon^{\frac{1}{1-\alpha}}-1 / 2\right)\left[\left\|u_{t}\right\|^{2}+\|u\|_{1, \Omega}^{2}\right] .
\end{aligned}
$$

Choosing $\varepsilon$ small enough that $C \varepsilon^{\frac{1}{1-\alpha}}<1 / 2$ we obtain

$$
(y(t))^{\frac{1}{1-\alpha}} \leq C\left[\|u(t)\|_{p+1}^{p+1}+|u(t)|_{k+1}^{k+1}\right] .
$$

which proves that the potential energy blows up. Moreover, $|u|_{H^{1}(\Omega)} \rightarrow \infty$. Indeed, assume that $|u|_{H^{1}(\Omega)}<M$. This implies that $\|u\|_{p+1}<M$ and $|u|_{k+1}<M$ (by the respective Sobolev embeddings), which is a contradiction.

So the final constraints on the parameters are:

$p, k>1, k>q, p>m, \alpha=\min \left\{\frac{1}{2}, \frac{1}{m}, \frac{1}{q}, \frac{p-m}{m(p+1)}, \frac{k-q}{q(k+1)}, \frac{p-1}{2(p+1)}\right\}$.

This proves the first statement of the theorem (when $k>q, p>m$ ).

Proof of the second statement: case $p>\max \{m, 2 q-1\}$. If $p+1>2 q$, and no restrictions on $k$ are imposed, the solution blows up with the help of internal source only. In order to prove this case, we need to modify the arguments in Lemma 4.3, so that the condition $k \geq q$ is no longer used.

We take up our proof at the level of (17):

$$
\begin{aligned}
\left|\int_{\Gamma} g\left(u_{t}\right) u d \Gamma\right| & \leq C\left(H^{\prime}(t)\right)^{\frac{q}{q+1}}|u(t)|_{q+1} \\
& =C\left(H^{\prime}(t)\right)^{\frac{q}{q+1}}(B(p, k, u))^{\frac{-\alpha q}{q+1}}(B(p, k, u))^{\frac{\alpha q}{q+1}}|u(t)|_{q+1} \\
& \leq C_{\delta_{2}} H^{\prime}(t)(H(t))^{-\alpha}+\delta_{2}(B(p, k, u))^{\alpha q}|u(t)|_{q+1}^{q+1} \\
& \leq C_{\delta_{2}} H^{\prime}(t)(H(t))^{-\alpha}+\delta_{2}(B(p, k, u))+\delta_{2}|u(t)|_{q+1}^{\frac{q+1}{1-\alpha q}} .
\end{aligned}
$$

We need to estimate the last boundary term in (30). We begin with the following estimates secured by trace and interpolation theorems of Besov space theory: 
- Trace theorem:

$$
|u|_{q+1} \leq C|u|_{W_{q+1}^{s}(\Omega)}, \quad s>\frac{1}{q+1} .
$$

- Interpolation theorem [34]:

$$
\left[H^{1}(\Omega), L_{p+1}(\Omega)\right]_{\theta}=W_{r}^{1-\theta}(\Omega), \quad r=\frac{2(p+1)}{(1-\theta)(p+1)+2 \theta},
$$

where the interpolation parameter is $\theta \in[0,1]$.

Set

$$
1-\theta \equiv \frac{1}{(q+1)(1-\alpha q)}>\frac{1}{q+1}
$$

for any $\alpha \in(0,1 / q)$, hence

$$
\theta=\frac{q}{q+1}-\frac{\alpha q}{(q+1)(1-\alpha q)} .
$$

After assuming

$$
p+1>2 q
$$

we obtain

$$
\frac{2(p+1)}{(1-\theta)(p+1)+2 \theta} \geq q+1
$$

as long as $\frac{\alpha q}{1-\alpha q}<\frac{p+1-2 q}{p-1}$.

Hence, with $p+1>2 q$, by the trace-interpolation-moment inequality we have

$$
|u|_{q+1} \leq C|u|_{W_{q+1}^{1-\theta}(\Omega)} \leq C|u|_{W_{\frac{2(p+1)}{(1-\theta)(p+1)+2 \theta}}^{1-\theta}} \leq C\|u\|_{1, \Omega}^{1-\theta}\|u\|_{p+1}^{\theta} .
$$

Consequently,

$$
|u|_{q+1}^{\frac{q+1}{1-\alpha q}} \leq C\|u\|_{1, \Omega}^{(1-\theta) \frac{q+1}{1-\alpha q}}\|u\|_{p+1}^{\theta \frac{q+1}{1-\alpha q}} \leq C \delta\|u\|_{1, \Omega}^{r(1-\theta) \frac{q+1}{1-\alpha q}}+C_{\delta}\|u\|_{p+1}^{\bar{r} \theta \frac{q+1}{1-\alpha q}}
$$

where in the last step Young's inequality is used with the conjugate exponents $r, \bar{r}$.

We take

$$
r \equiv \frac{2(1-\alpha q)}{(1-\theta)(q+1)}=2(1-\alpha q)^{2}<2
$$

Consequently,

$$
\bar{r}=\frac{2(1-\alpha q)^{2}}{2(1-\alpha q)^{2}-1}>1
$$

and recalling $\theta=\frac{q-\alpha q(q+1)}{(q+1)(1-\alpha q)}$, the exponent $\bar{r} \theta \frac{q+1}{1-\alpha \theta}=\frac{2 \theta(q+1)(1-\alpha q)}{2(1-\alpha q)^{2}-1}$ is required to satisfy

$$
\frac{2 \theta(q+1)(1-\alpha q)}{2(1-\alpha q)^{2}-1}=\frac{2(q-\alpha q(q+1))}{2(1-\alpha q)^{2}-1} \leq p+1 .
$$


The above is satisfied as long as $2 q \leq p+1, q<p$ and $\alpha$ is sufficiently small, so that $\frac{p+1}{2}(2 \alpha q)^{2}-(2 p-q+1)(2 \alpha q)+p+1-2 q \geq 0$. This last inequality is guaranteed by

$$
2 \alpha q \leq \frac{2 p+1-q-\sqrt{q^{2}+2 q+2 p^{2}-1}}{p+1}
$$

where

$$
\frac{2 p+1-q-\sqrt{q^{2}+2 q+2 p^{2}-1}}{p+1} \geq 0 \Leftrightarrow p \geq \max \{q, 2 q-1\} .
$$

Furthermore, since $2 q \leq p+1$ is equivalent to $\sqrt{q^{2}+2 q+2 p^{2}-1} \geq$ $(p+q)^{2}$, we can simplify $(31)$ to

$$
2 \alpha q \leq \frac{2 p+1-q-q-p}{p+1}=\frac{p+1-2 q}{p+1} .
$$

Thus, the final value for $p$ is subject to the following restrictions:

$$
p>\max \{1, q, 2 q-1\} \text {. }
$$

We note that when $\|u\|_{p+1} \leq 1$, Sobolev's embeddings yields the estimate $\|u\|_{p+1}^{t} \leq\|u\|_{1, \Omega}^{2}$ with any $t \geq 2$. Since in our case

$$
t=\frac{2(q-\alpha q(q+1))}{2(1-\alpha q)^{2}-1}
$$

we have $t \geq 2$ as long as $q \geq 2(1-\alpha q)^{2}+\alpha q(q+1)-1 \Leftrightarrow q \geq 2(1-\alpha q)-1$ for small $\alpha>0 \Leftrightarrow(q-1) / 2 \geq-\alpha q$ when $q \geq 1$.

Thus, it suffices to consider only large frequencies.

With such choice of parameters we have

$$
|u|_{q+1}^{\frac{q+1}{1-\alpha q}} \leq \delta\|u\|_{1, \Omega}^{2}+c_{\delta}\|u\|_{p+1}^{p+1}
$$

where the above estimate takes also in consideration both large and small frequencies. The inequality (32) leads to

LEMMA 4.4.

$$
\int_{\Gamma} g\left(u_{t}(t)\right) u(t) d \Gamma \leq C_{\delta_{2}} H^{\prime}(t)(H(t))^{-\alpha}+\delta_{2}\left[B(p, k, u)+C_{2}\|u(t)\|_{1, \Omega}^{2}\right] .
$$

Lemma 4.4 is a counterpart of Lemma 4.3. This allows one to complete the proof as before.

5. Proof of Theorem 3.2. The main difficulty in the proof is that the parameters $p$ and $k$ are not required to satisfy the constraints $p+1 \leq$ $2 n /(n-2)$ and $k+1 \leq(2 n-2) /(n-2)$. The above constraints allow one to control $L_{p+1}(\Omega)$ and $L_{k+1}(\Gamma)$ norms by $H^{1}(\Omega)$ norms. Thus, the main technical issue is to bypass Sobolev's embeddings. 
We proceed as before with the goal of proving a less demanding inequality

$$
y^{\prime}(t) \geq c(y(t))^{\frac{1}{1-\alpha}}-c_{0}
$$

for suitable positive constants $c, c_{0}$.

The main difference in the proof is at the level of small frequencies. Instead of using Sobolev's embeddings, in handling case 2 of small frequencies, we simply leave

$$
\|u(t)\|_{p+1} \leq c_{0}=1
$$

This leads to a counterpart of inequality (16), which now reads

$$
\|u(t)\|_{p+1}^{\frac{m+1}{1-\alpha m}} \leq C_{\Omega}[B(p, k, u)+M]
$$

for some positive constant $M$ equal to $c_{2}$ in (11), which does not depend on the initial energy $E(0)$.

This produces an interior damping counterpart of Lemma 4.2:

Lemma 5.1. There exists a constant $M>0$ such that

$$
\int_{\Omega} u g_{0}\left(u_{t}\right) d \Omega \leq C_{\delta_{1}} H^{\prime}(t)(H(t))^{-\alpha}+\delta_{1}\left[B(p, k, u)+C_{1}(H(0))\|u\|_{1, \Omega}^{2}+M\right] .
$$

Similarly in the case of boundary damping: We estimate small frequencies for case 2 as

$$
|u(t)|_{k+1} \leq c_{0}=1
$$

which then leads to

$$
|u(t)|_{k+1}^{\frac{q+1}{1-\alpha q}} \leq C_{\Omega}[B(p, k, u)+M] .
$$

Hence we obtain

Lemma 5.2. There exists a constant $M>0$ such that

$$
\begin{aligned}
\int_{\Gamma} g\left(u_{t}(t)\right) u(t) d \Gamma \leq & C_{\delta_{2}} H^{\prime}(t)(H(t))^{-\alpha} \\
& +\delta_{2}\left[B(p, k, u)+C_{2}(H(0))\|u(t)\|_{1, \Omega}^{2}+M\right] .
\end{aligned}
$$

The inequality for $y^{\prime}$ then becomes

Proposition 3.

$$
\begin{aligned}
y^{\prime}(t) \geq & {\left[(1-\alpha)-\varepsilon C_{\delta}\right] H^{\prime}(t)(H(t))^{-\alpha}+\varepsilon(2+\beta / 2)\left\|u_{t}(t)\right\|^{2} } \\
& +\varepsilon(\beta / 4)\|u(t)\|_{1, \Omega}^{2}+(\lambda \varepsilon / 2) B(p, k, u)+\varepsilon(2+\beta) H(t)-\epsilon M
\end{aligned}
$$

for some positive constant $M>0$.

The estimate for the error term $F(t)$ takes the form

$$
(F(t))^{\frac{1}{1-\alpha}} \leq C\left[\left\|u_{t}\right\|^{2}+\int_{\Omega}|u|^{p+1}\right]+M
$$

where $M$ results also from low frequencies estimate in case 2 at the end of the proof. 
The estimate from below for $H(t)$ becomes

$$
\begin{aligned}
H(t) & \geq c y^{\frac{1}{1-\alpha}}-C \varepsilon^{\frac{1}{1-\alpha}}(F(t))^{\frac{1}{1-\alpha}} \\
& \geq c y^{\frac{1}{1-\alpha}}-C \varepsilon^{\frac{1}{1-\alpha}}\left[\left\|u_{t}\right\|^{2}+\|u\|_{1, \Omega}^{2}+B(p, k, u)-M\right] .
\end{aligned}
$$

Combining all the estimates gives an inequality of the form

$$
y^{\prime}(t) \geq c(y(t))^{\frac{1}{1-\alpha}}-c_{0}
$$

with $0<1-\alpha<1$ and some constants $c>0, c_{0}$ (which can be traced back and does not depend on $E(0)$ - this fact is critical). Solutions to these inequalities blow up for initial conditions sufficiently positive (i.e. $H(0)>$ $M_{0}$ ), where $M_{0}>c_{0} c^{-1}$. The value of blow-up time can also be computed explicitly [32].

This can be seen as follows:

Let $\beta=1 /(1-\alpha)>1$. Then the inequality becomes

$$
y^{\prime}(t) \geq c(y(t))^{\beta}-c_{0}
$$

where $y(0) \geq M_{0}$. Our goal is to show that there exists $T<\infty$ such that $y(t) \rightarrow \infty$ as $t \rightarrow T^{-}$.

Choose $M_{0}>c_{0} / c$. This guarantees that $c y_{0}^{\beta}-c_{0}>0$. This implies that $c(y(t))^{\beta}-c_{0}>0$ for all $t \geq 0$. Now our claim is that

$$
c(y(t))^{\beta}-c_{0} \geq\left(c^{1 / \beta} y(t)-c_{0}^{1 / \beta}\right)^{\beta} .
$$

To see this, let $f(y)=c y^{\beta}-c_{0}-\left(c^{1 / \beta} y(t)-c_{0}^{1 / \beta}\right)^{\beta}$. Note that $f\left(\left(c_{0} / c\right)^{1 / \beta}\right)=0$. Then by the intermediate value theorem, we have

$$
f(y)-f\left(\left(c_{0} / c\right)^{1 / \beta}\right)=f^{\prime}(x)\left(y-\left(c_{0} / c\right)^{1 / \beta}\right) .
$$

Calculating $f^{\prime}(x)$, we obtain

$$
\begin{aligned}
f^{\prime}(x) & =c \beta x^{\beta-1}-\beta\left(c^{1 / \beta} x-c_{0}^{1 / \beta}\right)^{\beta-1} \cdot c^{1 / \beta} \\
& =\beta c^{1 / \beta}\left[c^{\beta-1 / \beta} \cdot x^{\beta-1}-\left(c^{1 / \beta} x-c_{0}^{1 / \beta}\right)^{\beta-1}\right] \geq 0
\end{aligned}
$$

since $c^{(\beta-1) / \beta} y^{\beta-1} \geq\left(c^{1 / \beta} y-c_{0}^{1 / \beta}\right)^{\beta-1} \Leftrightarrow c^{1 / \beta} y \geq c^{1 / \beta} y-c_{0}^{1 / \beta}$.

Now let $z(t)=c^{1 / \beta} y(t)-c_{0}^{1 / \beta}$. Then the inequality becomes $z^{\prime}(t) \geq$ $c^{1 / \beta}(z(t))^{\beta}$ with $z(0)>0$, whose solution blows up in finite time, i.e. there exists

$$
0<T=\frac{1}{(z(0))^{\beta}(\beta-1) c^{1 / \beta}}<\infty
$$

such that $z(t) \rightarrow \infty$ as $t \rightarrow T^{-}$. 
Acknowledgements. We would like to thank an anonymous referee for his valuable comments and suggestions. In particular, we are grateful for his remarks that led to a refinement of the range of parameters in the proof of Theorem 3.1.

\section{References}

[1] G. Avalos, Sharp regularity estimates for solutions to wave equations and their traces with prescribed Neumann data, Appl. Math. Optim. 15 (1997), 203-223.

[2] V. Barbu, Nonlinear Semigroups and Differential Equations in Banach Spaces, Noordhoff, 1976.

[3] V. Barbu, I. Lasiecka and M. A. Rammaha, On nonlinear wave equations with degenerate damping and source terms, Trans. Amer. Math. Soc. 357 (2005), 25712611.

[4] - , - - - Blow-up of generalized solutions to wave equations with nonlinear degenerate damping and source term, Indiana Univ. Math. J. 56 (2007), 995-1021.

[5] L. Bociu, Local and global wellposedness of weak solutions for the wave equation with nonlinear boundary and interior sources of supercritical exponents and damping, Nonlinear Anal., to appear.

[6] —, Existence, uniqueness and blow-up of solutions to wave equations with supercritical boundary/interior sources and damping, Ph.D. Thesis, Univ. of Virginia, 2008.

[7] L. Bociu and I. Lasiecka, Uniqueness of weak solutions for the semilinear wave equations with supercritical boundary/interior sources and damping, Discrete Contin. Dynam. Systems 22 (2008).

[8] S. Brenner and L. R. Scott, The Mathematical Theory of Finite Element Methods, Springer, 1994.

[9] M. Cavalcanti, V. N. Cavalcanti and P. Martinez, Existence and decay rates for the wave equation with nonlinear boundary damping and source term, J. Differential Equations 203 (2004), 119-158.

[10] I. Chueshov, M. Eller and I. Lasiecka, On the attractor for a semilinear wave equation with critical exponent and nonlinear boundary dissipation, Comm. Partial Differential Equations 27 (2002), 1901-1951.

[11] I. Chueshov and I. Lasiecka, Long time behavior of second order evolutions with nonlinear damping, Mem. Amer. Math. Soc. 195 (2008), no. 912.

[12] - - - Long time dynamics of a semilinear wave equation with nonlinear interior boundary damping and sources of critical exponents, in: Contemp. Math. 426, Amer. Math. Soc., 2007, 153-192.

[13] E. Feireisl, Global attractors for semilinear damped wave equations with supercritical exponent, J. Differential Equations 116 (1995), 431-447.

[14] V. Georgiev and G. Todorova, Existence of a solution of the wave equation with nonlinear damping and source terms, ibid. 109 (1994), 295-308.

[15] H. Koch and I. Lasiecka, Hadamard well-posedness of weak solutions in nonlinear dynamic elasticity - full von Karman systems, in: Progr. Nonlinear Differential Equations Appl. 50, Birkhäuser, 2002, 197-216.

[16] V. Komornik, Exact Controllability and Stabilization. Multipliers Method, Masson, Paris, 1994.

[17] J. Lagnese, Boundary Stabilization of Thin Plates, SIAM, Philadelphia, 1989. 
[18] I. Lasiecka, Mathematical Control Theory of Coupled PDE's, CBMS-SIAM Lecture Notes, SIAM, 2002.

[19] I. Lasiecka, J.-L. Lions and R. Triggiani, Nonhomogeneous boundary value problems for second order hyperbolic operators, J. Math Pures Appl. 65 (1986), 149-192.

[20] I. Lasiecka and J. Ong, Global solvability and uniform decays of solutions to quasilinear equation with nonlinear boundary dissipation, Comm. Partial Differential Equations 24 (1999), 2069-2107.

[21] I. Lasiecka and A. Stahel, The wave equation with semilinear Neumann boundary conditions, Nonlinear Anal. 15 (1990), 39-58.

[22] I. Lasiecka and D. Tataru, Uniform boundary stabilization of semilinear wave equations with nonlinear boundary damping, Differential Integral Equations 6 (1993), 507-533.

[23] I. Lasiecka and R. Triggiani, Trace regularity of the solutions of the wave equation with homogeneous Neumann boundary conditions and data supported away from the boundary, J. Math. Anal. Appl. 141 (1989), 49-71.

[24] -, - Regularity theory of hyperbolic equations with non-homogeneous Neumann boundary conditions. II, J. Differential Equations 94 (1991), 112-164.

[25] - - - Sharp regularity for mixed second order hyperbolic equations of Neumann type. Part I: The $L_{2}$-boundary case, Ann. Mat. Pura Appl. (4) 157 (1990), 285-367.

[26] —, - Control Theory for Partial Differential Equations: Continuous and Approximation Theories, Cambridge Univ. Press, 2000.

[27] H. Levine and L. E. Payne, Nonexistence theorems for the heat equation with nonlinear boundary conditions and for the porous medium equation backward in time, J. Differential Equations 16 (1974), 319-334.

[28] H. Levine and A. Smith, A potential well theory for the wave equation with a nonlinear boundary conditions, J. Reine Angew. Math. 374 (1987), 1-23.

[29] L. Payne and D. Sattinger, Saddle points and instability of nonlinear hyperbolic equations, Israel Math. J. 22 (1981), 273-303.

[30] D. R. Pitts and M. A. Rammaha, Global existence and non-existence theorems for nonlinear wave equations, Indiana Univ. Math. J. 51 (2002), 1479-1509.

[31] P. Radu, Weak solutions to the Cauchy problem of a semilinear wave equation with damping and source terms, Adv. Differential Equations 10 (2005), 1281-1300.

[32] M. Rammaha, private communication.

[33] M. A. Rammaha and T. A. Strei, Global existence and nonexistence for nonlinear wave equations with damping and source terms, Trans. Amer. Math. Soc. 354 (2002), 3621-3637.

[34] T. Runst and W. Sieckel, Sobolev Spaces of Fractional Order, Nemytskij Operators and Nonlinear Partial Differential Equations, de Gruyter, New York, 1996.

[35] R. Sakamoto, Mixed problems for hyperbolic equations, J. Math. Kyoto Univ. 10 (1970), 243-373.

[36] J. Serrin, G. Todorova and E. Vitillaro, Existence for a nonlinear wave equation with damping and source terms, Differential Integral Equations 16 (2003), 13-50.

[37] W. W. Symes, A trace theorem for solutions of the wave equation and the remote determination of acoustic sources, Math. Appl. Sci. 5 (1983), 131-152.

[38] D. Tataru, On the regularity of boundary traces for the wave equation, Ann. Scuola Norm. Sup. Pisa 6 (1998), 185-206.

[39] E. Vitillaro, Global existence of the wave equation with nonlinear boundary damping and source terms, J. Differential Equations 186 (2002), 259-298.

[40] -, A potential well theory for the wave equation with nonlinear source and boundary damping terms, Glasgow Math. J. 44 (2002), 375-395. 
[41] E. Vitillaro, Global nonexistence theorem for a class of evolution equations with dissipation, Arch. Ration. Mech. Anal. 149 (1999), 155-182.

[42] - Some new results on global nonexistence and blow-up for evolution problems with positive initial energy, Rend. Inst. Mat. Univ. Trieste 31 (2000), 245-275.

243 Avery Hall

University of Nebraska-Lincoln

Lincoln, NE 68588, U.S.A.

E-mail: lvb9b@virginia.edu
Department of Mathematics

University of Virginia

Charlottesville, VA 22904, U.S.A.

E-mail: il2v@virginia.edu

Received on 20.6.2008;

revised version on 30.7.2008 